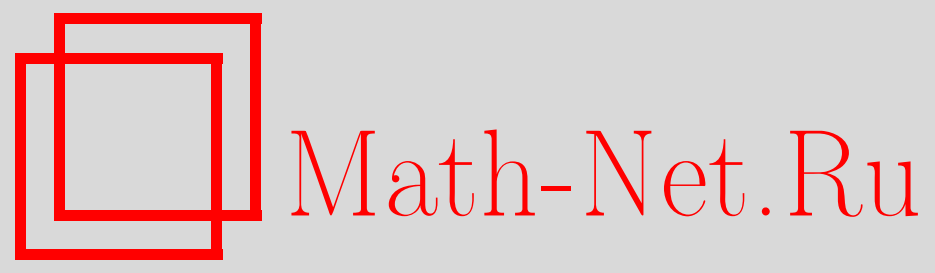

С. Б. Гашков, И. С. Сергеев, О применении метода аддитивных цепочек к инвертированию в конечных полях, Дискрет. матем., 2006, том 18, выпуск 4, 56-72

DOI: https://doi.org/10.4213/dm80

Использование Общероссийского математического портала Math-Net.Ru подразумевает, что вы прочитали и согласны с пользовательским соглашением http://www.mathnet.ru/rus/agreement

Параметры загрузки:

IP : 3.85 .5 .30

26 апреля 2023 г., 14:54:48 


\title{
О применении метода аддитивных цепочек к инвертированию в конечных полях
}

\author{
() 2006 г. . Б. Гашков, И. С. Сергеев
}

\begin{abstract}
Получены оценки сложности и глубины булевых схем для инвертирования в нормальных и полиномиальных базисах конечных полей. В частности, показано, что для инвертирования в нормальном базисе поля $G F\left(2^{n}\right)$ можно построить булеву схему со сложностью не более $(\lambda(n-1)+(1+o(1)) \lambda(n) / \lambda(\lambda(n))) M(n)$ и глубиной не более $(\lambda(n-1)+2) D(n)$, где $M(n), D(n)$ - соответственно сложность и глубина схемы для умножения в этом базисе, а $\lambda(n)=\left\lfloor\log _{2} n\right\rfloor$.

Работа выполнена при поддержке Российского фонда фундаментальных исследований, проект 05-01-00994, программой Президента Российской Федерации поддержки ведущих научных школ, грант НШ 5400.2006.1, и программой фундаментальных исследований Отделения математических наук РАН «Алгебраические и комбинаторные методы математической кибернетики», проект «Синтез и сложность управляющих систем».
\end{abstract}

\section{1. Введение}

Вопросы эффективной реализации арифметических операций в больших конечных полях характеристики два имеют существенное значение в криптографических приложениях (см., например, [1]). В [2, 3] показано, что инвертирование в поле $G F\left(2^{n}\right)$ может выполняться булевой схемой глубины $O(\log n)$ и сложности $n^{O(1)}$, однако константы в знаках $O(1)$ достаточно велики, что делает эти методы непригодными для практического применения.

Многие используемые на практике методы инвертирования в таких полях сводят задачу инвертирования к возведению в степень с показателем вида $2^{n}-1$. Действительно, для любого $x \in G F\left(2^{n}\right)^{*}$ (то есть $x \in G F\left(2^{n}\right)$ и $x \neq 0$ ) справедливо тождество Ферма

$$
x^{-1}=x^{2^{n}-2}=\left(x^{2}\right)^{2^{n-1}-1} .
$$

Задача возведения в степень может быть сформулирована в терминах аддитивных цепочек (об аддитивных цепочках см. [4], раздел 4.6.3).

Мы покажем, что наилучшим в определенном классе методов инвертирования в поле $G F\left(2^{n}\right)$ является метод Брауэра (1939 г), сводящийся к построению кратчайшей аддитивной цепочки для $n-1$. Необходимые цепочки для первых нескольких миллионов значений $n$ давно подсчитаны (в частности, при построении цепочек для $n \leqslant 1000$ можно пользоваться рекуррентным правилом из [4], с. 530). 
Идея применения аддитивных цепочек к задачам возведения в степень и инвертирования высказывалась явно во многих работах, и во многих работах использовалась неявно. В [5] отмечено, что метод инвертирования [6] является частным случаем метода Брауэра. Однако эта работа не очень проста для чтения и, по-видимому, малоизвестна (мы узнали о ее существовании уже в процессе подготовки нашей статьи). Во всяком случае, в выполненных позже работах $[7,8]$ нет упоминаний о методе Брауэра, а полученные там результаты фактически им перекрываются. Далее мы приводим простое построение схем для инвертирования в конечных полях, основанное на использовании методов Брауэра и Яо [9].

\section{2. Аддитивные цепочки и метод Брауэра}

Аддитивной цепочкой для числа $n$ называется любая начинаюшаяся с 1 последовательность натуральных чисел $a_{0}=1, a_{1}, \ldots, a_{m}=n$, в которой каждое число является суммой каких-то двух предыдущих чисел (возможно совпадающих), то есть для всех $i \geqslant 1$ выполнено равенство $a_{i}=a_{j}+a_{k}, j, k<i$. Под длиной цепочки $a_{0}, a_{1}, \ldots, a_{m}$ понимается число $m$. Через $l(n)$ обозначим длину кратчайшей аддитивной цепочки для $n$.

Возведение в степень может быть интерпретировано как построение аддитивной цепочки для показателя степени. Удвоения в аддитивной цепочке соответствуют возведениям в квадрат, а прочие сложения - умножениям.

Адлитивная цепочка называется линейной, если каждый ее элемент равен сумме предыдущего элемента и какого-то еще, то есть для всех $i \geqslant 1$ выполнено равенство $a_{i}=a_{i-1}+a_{j}, j<i$. Длина кратчайшей линейной цепочки обозначается через $l^{*}(n)$.

Метод Брауэра (см., например, [4], раздел 4.6.3) позволяет по заданной линейной цепочке $1, a_{1}, \ldots, a_{m}=n$ построить аддитивную цепочку для $2^{n}-1$ следующим образом. Выписывается последовательность

$$
1=2^{1}-1,2^{a_{1}}-1, \ldots, 2^{a_{m}}-1=2^{n}-1,
$$

затем в промежутки между числами вставляются последовательности удвоений. Так, между соседними числами $2^{a_{i}}-1$ и $2^{a_{i+1}}-1$, где $a_{i+1}=a_{i}+a_{j}$, помещается последовательность

$$
2\left(2^{a_{i}}-1\right), 2^{2}\left(2^{a_{i}}-1\right), \ldots, 2^{a_{i+1}-a_{i}}\left(2^{a_{i}}-1\right)=2^{a_{i+1}}-2^{a_{j}} .
$$

Поскольку

$$
2^{a_{i+1}}-1=\left(2^{a_{i+1}}-2^{a_{j}}\right)+\left(2^{a_{j}}-1\right)
$$

итоговая последовательность является линейной аддитивной цепочкой длины $n+m-1$, состоящей из $n-1$ удвоений и $m$ сложений.

Метод Брауэра можно использовать и с произвольной цепочкой для $n$. При этом, если $a_{i}=a_{j}+a_{k}$, то для вычисления $2^{a_{i}}-1$ последовательность удвоений необходимо вести от $2^{a_{j}}-1$ или от $2^{a_{k}}-1$. Число сложений в построенной цепочке также будет совпадать с длиной исходной цепочки для $n$, но число удвоений может быть больше, чем $n-1$. Цепочки, для которых методом Брауэра строится цепочка ровно с $n-1$ удвоениями, называются цепочками Ханзена (линейные цепочки являются частным случаем цепочек Ханзена, см. [4]). В цепочке, построенной методом Брауэра с исходной цепочкой Ханзена, все нелинейные шаги являются удвоениями. 
Из метода Брауэра следует, что

$$
l^{*}\left(2^{n}-1\right) \leqslant n+l^{*}(n)-1 .
$$

Это утверждение обобщается также на цепочки Ханзена, но гипотеза Шольца-Брауэра, утверждающая, что верно неравенство

$$
l\left(2^{n}-1\right) \leqslant n+l(n)-1
$$

по-видимому, до сих пор не доказана и не опровергнута. Также, видимо, не доказано неравенство

$$
l(n) \geqslant \lambda(n)+\lambda(v(n)),
$$

где $\lambda(n)=\left\lfloor\log _{2} n\right\rfloor$, а $v(n)-$ вес числа $n$, то есть количество единиц в его двоичной записи, хотя А. Шёнхаге в 1975 г. доказал (см. [4, 10]), что

$$
l(n) \geqslant \lambda(n)+\lambda(v(n))+O(1) .
$$

Известно (это тоже теорема Ханзена), что для любого $c$ существуют $\boldsymbol{n}$ такие, что $l(n)<l^{*}(n)-c$, но практически требование линейности не накладывает существенных ограничений, поскольку для всех $n \leqslant 12508$ справедливо равенство $l(n)=l^{*}(n)$, то есть для разумных значений $n$ всегда существует линейная аддитивная цепочка минимальной длины (см. [4]).

Метод Брауэра сводит задачу инвертирования в поле $G F\left(2^{n}\right)$ к построению кратчайшей аддитивной цепочки для $n-1$. Длина этой цепочки соответствует числу сложений при вычисления $2^{n}-2$, которое, в свою очередь, соответствует числу выполняемых умножений при возведении элемента поля в $\left(2^{n}-2\right)$-ю степень. Число возведений в квадрат при этом гораздо больше и, по меньшей мере, равно $n-1$. Однако возведение в квадрат в поле $G F\left(2^{n}\right)$ существенно проще умножения, и при выборе стандартного базиса, соответствующего неприводимому трехчлену или пятичлену, имеет сложность $O(n)$. Но еще удобнее при применения метода Брауэра в поле $G F\left(2^{n}\right)$ пользоваться нормальными базисами, так как возведение в квадрат в нормальном базисе практически не требует вычислительных затрат (осуществляется посредством циклического сдвига коэффициентов, см., например, [6]). Если через $M\left(G F\left(2^{n}\right)\right)$ обозначить сложность умножения в нормальном базисе поля $G F\left(2^{n}\right)$, то сложность метода Брауэра для инвертирования в поле $G F\left(2^{n}\right)$ можно оценить как $l(n-1) M\left(G F\left(2^{n}\right)\right)$.

В частности, для сложности $I\left(G F\left(2^{n}\right)\right)$ инвертирования в поле $G F\left(2^{n}\right)$ справедливы следующие оценки:

$$
I\left(G F\left(2^{n}\right)\right) \leqslant \begin{cases}9 M\left(G F\left(2^{n}\right)\right), & n \leqslant 127, \\ 10 M\left(G F\left(2^{n}\right)\right), & n \leqslant 191, \\ 11 M\left(G F\left(2^{n}\right)\right), & n \leqslant 379, \\ 12 M\left(G F\left(2^{n}\right)\right), & n \leqslant 607, \\ 13 M\left(G F\left(2^{n}\right)\right), & n \leqslant 1087,\end{cases}
$$

и т. д., но для некоторых конкретных значений $n$ оценки реально оказываются лучше.

Далее проводится сравнение общего метода Брауэра, применяемого к аддитивным цепочкам различных типов, с методами $[7,8]$. Обозначение $\left[i_{k-1}, i_{k-2}, \ldots, i_{0}\right]$ будет применяться для записи числа $i=i_{k-1} 2^{k-1}+i_{k-2} 2^{k-2}+\ldots+i_{0}$ в двоичной системе счисления (все $\left.i_{j} \in\{0,1\}\right)$. 


\section{3. Бинарный алгоритм и метод Ито-Цуйи}

Пусть $n=\left[n_{k-1}, n_{k-2}, \ldots, n_{0}\right]$. Используя схему Горнера, можно записать формулу

$$
n=\left(\ldots\left(2 n_{k-1}+n_{k-2}\right) 2+\ldots+n_{1}\right) 2+n_{0},
$$

по которой выписывается универсальная аддитивная цепочка для числа $n$ (вычисления производятся слева направо) $a_{0}=n_{k-1}=1, a_{1}=2 a_{0}=2, a_{2}=a_{1}+n_{k-2}, a_{3}=2 a_{2}$, $\ldots, a_{2 k-4}=a_{2 k-5}+n_{1}, a_{2 k-3}=2 a_{2 k-4}, a_{2 k-2}=a_{2 k-3}+n_{0}$. Удалив из построенной цепочки повторяющиеся элементы, получим окончательно цепочку, соответствующую так называемому бинарному (перебирающему разряды слева направо) алгоритму [4].

Чтобы сразу избежать повторений, можно воспользоваться следующим правилом. Удалим из двоичной записи числа $n$ единицу в старшем разряде, перед остальными единицами вставим двойки, а все нули заменим на двойки. В итоге получится слово из символов 1 и 2, в котором единицы означают прибавление 1, а двойки - удвоение. Например, для числа $n=19=[10011]$ получается слово 222121, которому соответствует аддитивная цепочка $1,2,4,8,9,18,19$.

Длина бинарной аддитивной цепочки для числа $n$ равна $\lambda(n)+v(n)-1$. Цепочка строится при помощи $\lambda(n)$ удвоений и $v(n)-1$ прочих сложений (в данном случае, прибавлений единицы). Таким образом, построенная на основе данной аддитивная цепочка для числа $2^{n}-1$ имеет длину $n+\lambda(n)+v(n)-2$, и содержит $\lambda(n)+v(n)-1$ сложений.

Такой же результат дает метод [6], предложенный в 1988 г. Ито и Цуйи (и с тех пор неоднократно цитируемый, см., например, [1]). Способ построения аддитивной цепочки по методу Ито-Цуйи основан на представлении

$$
2^{n}-1=\left(2^{2^{k-1}}-1\right) 2^{\left[n_{k-2}, \ldots, n_{0}\right]}+2^{\left[n_{k-2}, \ldots, n_{0}\right]}-1 .
$$

Продолжая раскладывать $2^{\left[n_{k-2}, \ldots, n_{0}\right]}-1$ и т. д., получаем тождество

$$
\begin{aligned}
2^{n}-1=\left(2^{2^{k-1}}-1\right) 2^{\left[n_{k-2}, \ldots, n_{0}\right]}+n_{k-2}\left(2^{2^{k-2}}-1\right) 2^{\left[n_{k-3}, \ldots, n_{0}\right]} & +\ldots \\
& +n_{1}\left(2^{2^{1}}-1\right) 2^{n_{0}}+n_{0},
\end{aligned}
$$

поскольку $2^{n_{0}}-1=n_{0}$. Воспользуемся тем, что

$$
2^{2^{l}}-1=(2+1)\left(2^{2}+1\right) \ldots\left(2^{2^{l-1}}+1\right),
$$

и приведем выражение (1) к виду

$$
\begin{aligned}
2^{n}-1=\left(\ldots \left(( ( 2 ^ { 2 ^ { k - 2 } } + 1 ) 2 ^ { n _ { k - 2 } 2 ^ { k - 2 } } + n _ { k - 2 } ) \left(2^{2^{k-3}}+\right.\right.\right. & 1) 2^{n_{k-3} 2^{k-3}} \\
& \left.\left.+n_{k-3}\right) \ldots\right)(2+1) 2^{n_{0}}+n_{0} .
\end{aligned}
$$

Формула записана так, что вычисления должны производиться слева направо. Аналогично выпишем формулу, которой следует метод Брауэра с использованием бинарной аддитивной цепочки для $n$ (для краткости, в дальнейшем будем называть его просто бинарным алгоритмом в том случае, когда имеется в виду построение цепочки для $2^{n}-1$ )

$$
\begin{array}{r}
2^{n}-1=\left(\ldots\left(\left(2^{n_{k-1}}+1\right) 2^{n_{k-2}}+n_{k-2}\right)\left(2^{\left[n_{k-1}, n_{k-2}\right]}+1\right) 2^{n_{k-3}}\right. \\
\left.\left.\quad+n_{k-3}\right) \ldots\right)\left(2^{\left[n_{k-1}, \ldots, n_{1}\right]}+1\right) 2^{n_{0}}+n_{0} .
\end{array}
$$


Отсюда видно, что два указанных метода различаются лишь порядком выполнения операций. Для большей наглядности выпишем формулы для первого и второго методов для конкретного значения $n=19=[10011]$ :

$$
\begin{aligned}
& 2^{19}-1=\left(\left(2^{8}+1\right)\left(2^{4}+1\right)\left(2^{2}+1\right) 2^{2}+1\right)(2+1) 2+1, \\
& 2^{19}-1=\left((2+1)\left(2^{2}+1\right)\left(2^{4}+1\right) 2+1\right)\left(2^{9}+1\right) 2+1,
\end{aligned}
$$

где жирным шрифтом выделены единицы, соответствующие разрядам числа 19.

Укажем другой способ построения бинарной цепочки для $n$, не использующий схемы Горнера. Она получается удалением повторяющихся элементов из цепочки $1,2,\left[n_{1}, n_{0}\right], 4,\left[n_{2}, n_{1}, n_{0}\right], \ldots,\left[n_{k-2}, \ldots, n_{0}\right], 2^{k-1},\left[n_{k-1}, \ldots, n_{0}\right]$ и также использует $\lambda(n)$ удвоений и $v(n)-1$ прочих сложений - некоторые удвоения являются нелинейными шагами.

\section{4. Метод множителей}

О методе множителей и, как следствие, о существовании для некоторых $n$ цепочек, более коротких, чем бинарные, было известно еще в XIX веке (см. [4]).

Пусть $n=s t$ и построены аддитивные цепочки для $s$ и $t$

$$
1, a_{1}, \ldots, a_{i}=s ; \quad 1, b_{1}, \ldots, b_{j}=t .
$$

Тогда для $n$ выписывается аддитивная цепочка

$$
1, a_{1}, \ldots, a_{i}=s, s b_{1}, s b_{2}, \ldots, s b_{j}=n,
$$

длина которой равна сумме длин аддитивных цепочек для сомножителей. Если для вычисления $s$ и $t$ используются бинарные цепочки, то длина цепочки для $n$ равна $\lambda(s)+\lambda(t)+v(s)+v(t)-2$. При $n=15$ метод множителей впервые улучшает результат бинарного метода (с 6 до 5).

Применительно к построению цепочки для $2^{n}-1$ метод множителей означает, что в вычислениях используется разложение

$$
2^{n}-1=\left(2^{s}-1\right)\left(2^{s(t-1)}+2^{s(t-2)}+\ldots+2^{s}+1\right) .
$$

Цепочка для первого сомножителя строится одним из двух методов предыдущего параграфа. Теперь заметим, что если в равенствах (2), (3) заменить в левой части $2^{n}-1$ на $2^{n-1}+2^{n-2}+\ldots+1$, а затем в обеих частях двойки в основаниях заменить на $2^{s}$, то формулы останутся справедливыми. Таким образом мы можем построить цепочку для $2^{s(t-1)}+2^{s(t-2)}+\ldots+2^{s}+1$, используя $s(t-1)$ удвоений и $\lambda(t)+v(t)-1$ сложений. Так как в цепочке для $2^{s}-1$ используются $s-1$ удвоений и $\lambda(s)+v(s)-1$ сложений, окончательно получаем цепочку для $2^{n}-1$, состоящую из $n-1$ удвоений и $\lambda(s)+\lambda(t)+v(s)+v(t)-2$ сложений. Отметим также, что если в качестве базового здесь выбирается бинарный метод, то итоговая формула для вычисления $2^{n}-1$ совпадает с формулой, построенной сразу по аддитивной цепочке (4) для $n$.

Приведем пример для $n=15, s=3, t=5$ :

$$
2^{15}-1=((2+1) 2+1)\left(\left(2^{3}+1\right)\left(2^{6}+1\right) 2^{3}+1\right) .
$$

Точно в таком виде метод множителей был фактически переоткрыт в [7]. Естественно, в качестве базового метода выбирался метод Ито-Цуйи. 


\section{Метод множителей и метод Такаги-Ёшики-Такаги}

В вышеизложенном виде метод множителей применим только к составным числам $n$, но в случае, когда $n$ - простое число, цепочку для $n$ можно получить из цепочки для $n-1$ присоединением одного элемента. Для небольших $n$ хорошо себя зарекомендовало следующее правило (обобщенный метод множителей):

(1) если $n$ - составное число, то применяется обычный метод множителей к разложению $n=p q$, где $p-$ наименьший простой делитель $n$.

(2) в противном случае, если $n-$ простое число, перейти к $n-1$.

Это правило может быть применено рекурсивно к получающимся в разложении сомножителям.

Известно, что первый шаг указанного обобщенного метода множителей неоптимален лишь для ста пяти значений $n$ из диапазона $[2,1000]$. Более точно, значения $l(n)$ в этом диапазоне могут быть вычислены посредством формулы, приведенной на с. 530 в [4],

$$
l(n)=\min \left\{l(n-1)+1, l_{n}\right\}-\delta_{n},
$$

где $l_{n}=\infty$ при простом $n$ и $l_{n}=l(p)+l(q)$ при $n=p q$, причем $p-$ минимальный простой делитель $n$, а $\delta_{n}=0$ во всех случаях, кроме ста пяти значений $n$, для которых $\delta_{n}=1$ (список таких $n$ приведен на с. 531 в [4]).

Вскоре после появления этого алгоритма Такаги, Ёшики и Такаги в [7] предложили его обобщение (ТҮТ-алгоритм), подобное обобщенному методу множителей.

В основе ТҮТ-алгоритма лежит тот факт, что если $n=p q+h, h \geqslant 0$, то для $n$ может быть построена аддитивная цепочка длины $l_{p}+l_{q}+h$, где $l_{p}$ и $l_{q}$ - длины аддитивных цепочек для $p$ и $q$. Авторы [7] ведут изложение не в терминах аддитивных цепочек, здесь мы даем переформулировку описания их алгоритма. Цепочка для $p q$ строится по методу множителей, затем к ней дописываются члены $p q+1, p q+2, \ldots, p q+h=n$.

Таким образом, предлагается перебирать всевозможные представления $n$ в виде $p q+h$ и искать то, на котором достигается минимум $l_{p}+l_{q}+h$, где величины $l_{p}$ и $l_{q}$ получены тем же ТҮТ-алгоритмом (если дальнейшее разложение не производится, то методом ИтоЦуйи). Величина

$$
l_{n}=\min \left\{l_{p}+l_{q}+h \mid n=p q+h\right\}
$$

является точной оценкой сложности ТҮТ-алгоритма (то есть числа сложений в цепочке для $\left.2^{n}-1\right)$. Нетрудно заметить, что на самом деле

$$
l_{n}=\min \left\{\left\{l_{p}+l_{q} \mid n=p q\right\}, l_{n-1}+1\right\},
$$

(ср. с обобщенным методом множителей). В качестве базового выбирается алгоритм ИтоЦуйи. Аддитивная цепочка для $2^{n}-1$ строится по формуле

$$
2^{n}-1=\left(\ldots\left(\left(2^{p q}-1\right) 2+1\right) \ldots\right) 2+1 \text {, }
$$

где число дополнительных пар удвоений и прибавлений единиц равно $h$.

Для $n \leqslant 1000$ окончательное разложение (когда в соответствии с ТҮТ-алгоритмом выписаны разложения для сомножителей $p$ и q, и т. д.) обычно оказывается очень коротким. Так, для $n=383$ разложение выглядит так:

$$
383=(38 \times 5+1) \times 2+1,
$$


и это самое длинное из приведенных авторами разложений. В данном примере

$$
l_{383}=l_{38}+l_{5}+l_{2}+2=13,
$$

где $l_{38}=7, l_{5}=3, l_{2}=1$ из метода Ито-Цуйи.

Перебор получается не таким и большим из-за того, что $h$ входит в оценку сложности линейным образом, оно почти всегда равно 0 или 1 . Так, среди примеров оптимальных с точки зрения ТҮТ-алгоритма разложений в конце работы [7] лишь один раз встречается $h=2$ (для $n=863=41 \times 21+2$ ).

\section{5. ТYТ-алгоритм и метод Брауэра}

Заметим, что в оценках сложности ТҮТ-алгоритма ничего не изменится, если в качестве базового выбрать не метод Ито-Цуйи, а бинарный метод. Действительно, по имеющемуся ТҮТ-разложению для $n$ можно построить аддитивную цепочку так, как было указано выше, и применить общий метод построения цепочки для $2^{n}-1$ из цепочки для $n$, а это и будет эквивалентно по сложности применению ТҮТ-алгоритма. Например, в случае $n=863$ строятся бинарные цепочки $1,2,4,5,10,20,40,41$ и $1,2,4,5,10,20,21$, и окончательно цепочка $1,2,4,5,10,20,40,41,41 \cdot 2,41 \cdot 4,41 \cdot 5,41 \cdot 10,41 \cdot 20,41 \cdot 21=861$, 862,863 длины 15 , как и в ТҮТ-алгоритме.

Поэтому метод Такаги-Ёшики-Такаги никогда не превосходит метод Брауэра, который годится для произвольной линейной аддитивной цепочки (и вообще для любой цепочки, если количество удвоений несущественно), а не только для линейных цепочек специального вида, соответствующих ТҮТ-алгоритму. Более того, мы покажем, что алгоритм [7] в некоторых случаях уступает методу Брауэра.

Первое, что обращает на себя внимание - это способ построения цепочки для $2^{m+h}-1$, если $2^{m}-1$ уже вычислено (см. (5)), который требует $h$ дополнительных сложений. Ведь можно отдельно вычислить $2^{h}-1$ с помощью $l_{h}$ сложений и затем воспользоваться формулой

$$
2^{m+h}-1=\left(2^{m}-1\right) 2^{h}+\left(2^{h}-1\right)
$$

которая требует $l_{h}+1=O(\log h)$ дополнительных сложений, что дает выигрыш уже при $h=4$. Еще лучше использовать при построении цепочки для $2^{h}-1$ слагаемые, вычисляемые в цепочке для $2^{m}-1$.

Рассмотрим пример, показывающий, какое влияние это оказывает на сложность ТYТ-алгоритма. Из таблицы, приведенной в [7] для некоторых значений $n$, мы находим среди оптимальных разложений $767=59 \times 13$. Это значит, что число $n=59$ является неделимым с точки зрения ТҮТ-алгоритма (то есть сразу должен применяться метод Ито-Цуйи). При помощи бинарного метода легко проверяется, что $l_{59}=9$. Рассмотрим разложение $59=7 \times 8+3$, которое в ТҮТ-методе отсеивается в силу того, что $l_{7}+l_{8}+3=10>9$. Однако можно воспользоваться формулой

$$
2^{59}-1=\left(2^{56}-1\right) 2^{3}+\left(2^{3}-1\right)=\left(2^{56}-1\right) 2^{3}+2+5,
$$

согласно которой требуется два дополнительных сложения вместо трех, поскольку числа 2 и 5 встречаются в аддитивной цепочке для $2^{7}-1$, выписываемой методом Ито-Цуйи на первом шаге ТҮТ-алгоритма (см. (2)). Полученная цепочка имеет длину 9. А теперь применим бинарный метод в качестве базового. Он позволяет обойтись лишь одним дополнительным сложением, так как $2^{3}-1$ в бинарном методе вычисляется при построении 
цепочки для $2^{7}-1$. Цепочка для $n=59$, которая используется в том же самом разложении с базовым бинарным методом, $1,2,3,6,7,14,28,56,59$, имеет длину 8 , что доказывает неоптимальность ТҮТ-алгоритма.

Из этого примера можно увидеть, что, хотя разница между методом Ито-Цуйи и бинарным вариантом метода Брауэра кажется несущественной, однако бинарный метод лучше адаптирован к построению цепочек для разложений, используемых в ТҮТ-алгоритме, поскольку вычисляет достаточно много промежуточных членов вида $2^{k}-1$ (а именно, при каждом сложении).

Укажем еще примеры, когда ТYТ-алгоритм уступает методу Брауэра. В списке оптимальных разложений из [7] можно найти неделимые числа $k$ с весом $v(k) \geqslant 5$. Известно, что бинарный метод всегда оптимален для чисел с весом 3 и оптимален для чисел веса 4 , за исключением нескольких случаев (см., например, [4]), но, вообще говоря, неоптимален для чисел веса 5 и более. Так, среди примеров [7] было обнаружено четыре числа веса 5 , это $k=59,115,173,213$. Легко проверяется (например, используя сведения на с. 530 в [4]), что для всех этих чисел бинарная цепочка не является кратчайшей, следовательно, ТYТ-алгоритм неоптимален также при $n \in\{115,173,213\}$. Можно также заметить среди чисел, неделимых для ТYТ-алгоритма, числа 83 и 149 веса 4, обладающие цепочкой, более короткой, чем бинарная.

В табл. 1 приводятся значения чисел умножений в полях $G F\left(2^{n}\right)$ при $n \leqslant 300$ для инвертирования методом Брауэра в сравнении с ТҮТ-методом. Последний неоптимален уже при $n=24$, а при $n=348$ числа умножений, требуемых этими методами, различаются на 2.

\section{6. О глубине и сложности булевых схем для инвертирования}

Если мы хотим построить булеву схему для инвертирования из схем для умножения указанным выше методом, то представляет интерес задача минимизации глубины такой схемы (определение булевой схемы и ее глубины см., например, в [11]).

Минимальная глубина, очевидно, будет достигаться, когда глубина соответствующей аддитивной цепочки для $2^{n}-1$ будет минимальной. Игнорируя, как и выше, элементы удвоения, можно, повторяя доказательство теоремы Брауэра, получить, что глубина аддитивной цепочки для $2^{n}-1$ не превосходит глубины цепочки для числа $n$.

Очевидно, что минимальная глубина цепочки для $n$ равна $\left\lceil\log _{2} n\right\rceil$. Пример цепочки с такой глубиной и длиной $\lambda(n)+v(n)-1$ приведен в конце $\S 3$.

Например, для $n=27$ цепочкой минимальной длины 6 является цепочка $1,2,3,6$, $9,18,27$, но более длинная цепочка $1,2,3,4,8,11,16,27$ имеет меньшую глубину 5. По ней можно построить цепочку для вычисления $2^{27}-1$, в которой все неудваивающие шаги имеют вид $1,2^{2}-1,2^{3}-1,2^{4}-1,2^{8}-1,2^{11}-1,2^{16}-1,2^{27}-1$, и которая также имеет глубину 5 , если игнорировать удвоения.

Если для умножения в нормальном базисе применить метод Месси-Омура [12], то можно построить схему для умножения глубины $1+\left\lceil\log _{2}(2 n-1)\right\rceil$ и сложности $O\left(n^{3}\right)$. Поэтому можно построить в том же базисе схему для инвертирования глубины $\left\lceil\log _{2} n\right\rceil\left(1+\left\lceil\log _{2}(2 n-1)\right\rceil\right)$ и сложности $O\left(n^{3} \log n\right)[6]$. Для так называемых нормальных базисов низкой сложности (см., например, [13]) последняя оценка очевидно понижается до $O\left(n^{2} \log n\right)$. Используя метод сведения умножения в нормальных базисах к умножению в стандартном базисе (см. [13]), можно построить в любом нормальном базисе схему для 
Таблица 1. Число умножений для инвертирования в полях $G F\left(2^{n}\right)$

\begin{tabular}{|c|c|c|}
\hline 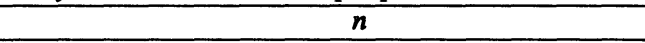 & TYT & Брауэр \\
\hline 45 & 2 & 2 \\
\hline 679 & 3 & 3 \\
\hline 810111317 & 4 & 4 \\
\hline $1214-16 \quad 18 \quad 19212533$ & 5 & 5 \\
\hline $20222326-2931343537414965$ & 6 & \multirow[t]{2}{*}{6} \\
\hline 24 & \multirow[b]{2}{*}{7} & \\
\hline $\begin{array}{l}30323638-404243454650-53555761666769 \\
738197129 \\
4447\end{array}$ & & 7 \\
\hline 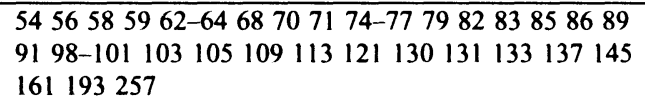 & 8 & \multirow[t]{2}{*}{8} \\
\hline 486078848793 & & \\
\hline 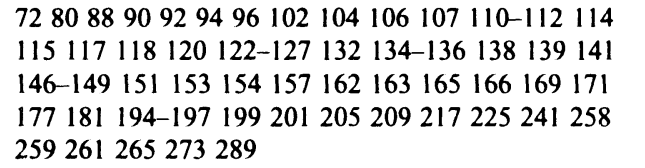 & 9 & \multirow[t]{2}{*}{9} \\
\hline 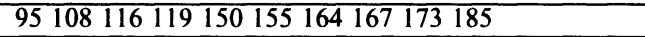 & \multirow[b]{2}{*}{10} & \\
\hline $\begin{array}{lllllllllll}128 & 140 & 142-144 & 152 & 156 & 158-160 & 170 & 172 & 175 & 176 \\
178 & 179 & 182-184 & 186-188 & 190 & 191 & 198 & 200 & 202 & 203 \\
206-208 & 210 & 211 & 213 & 218-223 & 226 & 227 & 229 & 232 & 233 \\
235 & 239 & 242-247 & 249 & 251 & 253 & 256 & 260 & 262 & 263 & 266 \\
267 & 269 & 271 & 274 & 275 & 277 & 281 & 290-293 & 295 & 297 & 298\end{array}$ & & \multirow[t]{2}{*}{10} \\
\hline 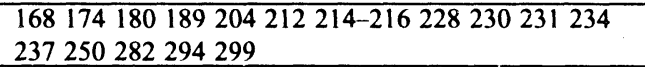 & \multirow[b]{2}{*}{11} & \\
\hline $\begin{array}{l}192 \quad 224236238240248252254255264268270 \\
272 \quad 276278-280283285-288296 \\
284300\end{array}$ & & 11 \\
\hline
\end{tabular}

умножения глубины $O(\log n)$ и сложности $O\left(n^{2} / \log n\right)$. Для этого достаточно перейти от нормального базиса к стандартному, что всегда можно сделать с помощъю схемы сложности $O\left(n^{2} / \log n\right)$ и глубины $\log n+O(1)$, реализующей соответствующее линейное преобразование (такие схемы можно построить методом О. Б. Лупанова [11]), выполнить умножение в стандартном базисе любым методом быстрого умножения и вернуться в исходный нормальный базис. Для некоторых нормальных базисов низкой сложности (например, оптимальных нормальных и вообще гауссовых нормальных базисов) можно доказать, что сложность умножения равна $O(n \log n \log \log n)$ при глубине $O(\log n)$ (см. $[5,13,14])$, однако такие базисы существуют не для всех $n$.

Из сказанного вытекает, что для инвертирования в нормальных базисах всегда можно построить схемы сложности $O\left(n^{2}\right)$ и глубины $O\left(\log ^{2} n\right)$, а для некоторых $n$ оценка сложности понижается до $O\left(n \log ^{2} n \log \log n\right)$ (с довольно большой мультипликативной константой в знаке $O$ ).

Метод, позволяющий строить для некоторых значений $n$ схемы глубины, меньшей, чем $\lambda^{2}(n)$, указан в [15]. 


\section{7. Об инвертировании в произвольных полях $G F\left(q^{n}\right)$}

В случае поля $G F\left(q^{n}\right), q>2$, согласно [6] для инвертирования можно вычислить $y=x^{\left(q^{n}-1\right) /(q-1)}$, заметить, что $y \in G F(q)$, так как $y^{q-1}=x^{q^{n}-1}=1$, и применить формулу

$$
x^{-1}=\left(x^{\left(q^{n-1}-1\right) /(q-1)}\right)^{q} y^{-1},
$$

справедливость которой проверяется путем сравнения степеней в обеих частях равенства

$$
-1=q\left(q^{n-1}-1\right) /(q-1)-\left(q^{n}-1\right) /(q-1) .
$$

Вычисление $y^{-1}$ и умножение на него при малых $q$ делается быстро, так как $y \in G F(q)$. Остается вычислить $x^{\left(q^{n-1}-1\right) /(q-1)}$. Предполагая, что возведение в степень $q$ делается быстро (это заведомо так при использовании нормальных базисов), и игнорируя эти операции, можно перенести доказательство теоремы Брауэра на этот случай и получить оценку для числа умножений при вычислении $x^{\left(q^{n-1}-1\right) /(q-1)}$ в том же виде $l(n-1)$, что и в случае $q=2$.

Для вычисления $y=x\left(x^{\left(q^{n-1}-1\right) /(q-1)}\right)^{q}$ нужна еще одна операция умножения, но более простая, чем обычное умножение, так как в ней надо вычислить только одну компоненту произведения, ведь $y \in G F(q)$.

Подобная схема используется при инвертировании в полях составной степени $G F\left(2^{n m}\right)$ (как, например, в [8]). Если $q=2^{m}$, то инвертирование в основном поле сводится к возведению в степень $\left(q^{n-1}-1\right) /(q-1)$ в поле $G F\left(q^{n}\right)$ и инвертированию в поле $G F(q)$. В [8] параметр $m$ выбирается небольшим (фактически рассматривают $m=4,8$ ), для возведения в степень $\left(q^{n-1}-1\right) /(q-1)$ используется ТҮТ-алгоритм. Так, представляя поля $G F\left(2^{384}\right)$ и $G F\left(2^{480}\right)$ как $G F\left(q^{96}\right)$ и $G F\left(q^{120}\right.$ ) (то есть $m=4$ ), авторы [8] получают сложность 11 умножений в обоих случаях, тогда как непосредственное применение даже метода Брауэра требует 12 умножений. Если же выбрать $m=8$ (что допускается) и рассматривать поля $G F\left(q^{48}\right)$ и $G F\left(q^{60}\right)$, то ТҮТ-алгоритм не приводит к уменьшению сложности. Однако, применение метода Брауэра в этих полях позволяет улучшить оценки [8] до 10.

В [5] для инвертирования в поле $G F\left(q^{n}\right), q>2$, применяется схема, не использующая инвертирования в $G F(q)$ и имеющая несколько большую сложность. Эта схема основана на использовании формулы

$$
x^{-1}=x^{\left(q^{n-1}-1\right) q} x^{q-2} .
$$

Вычисление $x^{q^{n-1}-1}$ проводится аналогично тому, как это было указано выше при использовании метода Брауэра.

\section{8. Быстрое инвертирование в полиномиальном базисе}

Используя для инвертирования в полиномиальном базисе поля $G F\left(q^{n}\right)$ быструю модификацию расширенного алгоритма Евклида совместно с алгоритмом Шёнхаге умножения многочленов (см., например, [10]), можно вычислить $g^{-1} \bmod f$ с помощью $O\left(n \log ^{2} n \log \log n\right)$ операций в поле $G F(q)$. Однако мультипликативный коэффициент в этой оценке велик (несколько сотен), и указанный алгоритм затруднительно реализовать в виде булевой схемы. Поэтому для практического применения лучше подходит, как и в случае нормальных базисов, метод Брауэра. При этом операция Фробениуса $g^{q^{m}} \bmod f$, согласно [14], может быть выполнена как модулярная композиция

3 Дискретная математика, т.18 №4 
$g(h) \bmod f$, где $h=x^{q^{m}} \bmod f$. Действительно, если

$$
g(x)=\sum_{i=0}^{s} a_{i} x^{i},
$$

To

$$
\begin{aligned}
g^{q^{m}}(x) & =\sum_{i=0}^{s} a_{i}^{q^{m}} x^{q^{m} i}=\sum_{i=0}^{s} a_{i} x^{q^{m}} \bmod f \\
& =\sum_{i=0}^{s} a_{i} h^{i} \bmod f=g(h) \bmod f
\end{aligned}
$$

Число операций в поле $G F(q)$, необходимых для выполнения модулярной композиции (см., например, [14]), равно

$$
S(n)=O\left(n^{(\omega+1) / 2}\right)+6 n^{1 / 2}(M(n)+O(n)),
$$

где $\omega$ - экспонента матричного умножения, наилучшая верхняя оценка для которой получена Виноградом и Купершмитом (см., например, [10]). Для схемной реализации множитель 6 в последнем слагаемом можно опустить. Главный член в этой оценке при малых $q$ и $M(n)=o\left(n^{\omega / 2}\right)$ есть $O\left(\left(n^{(\omega+1) / 2}\right)=O\left(n^{1,688}\right)\right.$, а с учетом результата Пана и Хуана о матричном умножении (см. также [10]) он равен $O\left(n^{1,667)}\right.$. Для глубины схем умножения матриц справедлива логарифмическая оценка (см., например, [16]). Однако мультипликативные константы во всех этих оценках, по-видимому, велики, и это обстоятельство, вероятно, делает указанный метод практически неприемлемым даже при $\boldsymbol{n}$ порядка 1000 .

На практике используются методы с худшей асимптотикой. Так, для $q=2$, основываясь на методе Штрассена (см., например, раздел 4.6.4 в [4]), можно построить схему для модулярной композиции с фиксированным многочленом сложности

$$
S(n)=O\left(n^{\log _{2} \sqrt{14}} /(\log n)^{\log _{2}(7 / 4)}\right) .
$$

Для $q>2$ оценка сложности имеет вид

$$
S(n)=O\left(n^{\log _{2} \sqrt{14}}\right) M(G F(q)) .
$$

Справедлива следующая теорема.

Теорема 1. Для инвертирования можно построить схему сложности

$$
I\left(G F\left(q^{n}\right)\right) \leqslant l(n-1) M\left(G F\left(q^{n}\right)\right)+n M(G F(q))+I(G F(q))+F\left(G F\left(q^{n}\right)\right)+l(n-1) S(n)
$$

u глубины

$$
O\left(\log ^{2} n\right) D_{M}(G F(q))+D_{F}\left(G F\left(q^{n}\right)\right)+D_{I}(G F(q)),
$$

где $D_{M}(G F(q))$ - глубина схемы умножения и $D_{I}(G F(q))$-глубина схемы для инвертирования в поле $G F(q)$, а $F\left(G F\left(q^{n}\right)\right)$ и $D_{F}\left(G F\left(q^{n}\right)\right)$ - сложность и глубина схемы для возведения в степень $q$ в поле $G F\left(q^{n}\right)$.

Для доказательства можно взять минимальную цепочку $1=a_{0}, \ldots, a_{r}=n-1$, $r=l(n-1)$, и построить цепочку для $\left(q^{n-1}-1\right) /(q-1)$, состоящую из чисел 
$\left(q^{a_{i}}-1\right) /(q-1)$, в промежутки между которыми вставлены результаты операции умножения на числа $q^{a_{j}}$ (аналогично случаю $q=2$, см. §2), в используемой аддитивной записи соответствующие преобразованиям Фробениуса, так как

$$
\left(q^{a_{i}}-1\right) q^{a_{j}} /(q-1)+\left(q^{a_{j}}-1\right) /(q-1)=\left(q^{a_{i}+a_{j}}-1\right) /(q-1) .
$$

Значит, для вычисления $x^{\left(q^{n-1}-1\right) /(q-1)}$ нужны операции Фробениуса для показателей $a_{0}, \ldots, \dot{a}_{r}$ (возможно, не все). Операция Фробениуса $g^{q^{m}} \bmod f$ может быть выполнена как модулярная композиция $g(h) \bmod f$, где $h=x^{q^{m}} \bmod f$. Всего нужно не более $l(n-1)$ модулярных композиций, если уже вычислены многочлены $h_{i}=x^{q^{a_{i}}} \bmod f$, $i=1, \ldots, r$.

Вычисление

$$
y=x\left(x^{\left(q^{n-1}-1\right) /(q-1)}\right)^{q}
$$

выполняется схемой сложности

$$
F\left(G F\left(q^{n}\right)\right)+M\left(G F\left(q^{n}\right)\right),
$$

а вычисление

$$
x^{-1}=y^{-1}\left(x^{\left(q^{n-1}-1\right) /(q-1)}\right)^{q}
$$

выполняется схемой сложности $I(G F(q))+n M(G F(q))$, так как $\left(x^{\left(q^{n-1}-1\right) /(q-1)}\right)^{q}$ уже вычислено, а умножение элемента поля $G F\left(q^{n}\right)$ на элемент поля $G F(q)$ выполняется со сложностью $n M(G F(q))$.

Сложность предвычислений (если имеется в виду программная реализация) можно оценить как

$$
l(n-1) S(n)+F\left(G F\left(q^{n}\right)\right) .
$$

Действительно, поскольку $a_{i}=a_{j}+a_{k}$,

$$
\begin{aligned}
h_{i} & =x^{q^{a_{i}}} \bmod f=x^{q^{a_{j}+a_{k}}} \bmod f=\left(x^{q^{a_{j}}}\right)^{q^{a_{k}}} \bmod f \\
& =\left(x^{q^{a_{j}}} \bmod f\right)^{q^{a_{k}}} \bmod f=h_{j}^{q^{a_{k}}} \bmod f=h_{j}\left(h_{k}\right) \bmod f
\end{aligned}
$$

и для вычисления коэффициентов многочленов $h_{i}=x^{q^{a_{i}}} \bmod f, i=1, \ldots, r$, достаточно вычислить $x^{q} \bmod f$ и выполнить $r=l(n-1)$ модулярных композиций.

\section{Уточнения в случае $q=2$}

Выбирая вместо минимальной цепочки обычную линейную бинарную цепочку, состоящую из $v(n)$ прибавлений единицы и $\lambda(n)-1$ удвоений (в отличие от $\S 3$, считаем, что элемент 2 цепочки получается из 1 прибавлением единицы), получаем следующий результат.

Теорема 2. Можно построчть схему для инвертирования в полиномиальном базисе сложности

$$
\begin{aligned}
I\left(G F\left(2^{n}\right)\right) \leqslant(\lambda(n-1)+v(n-1)-1) M( & \left.G F\left(2^{n}\right)\right) \\
& +v(n-1) K\left(G F\left(2^{n}\right)\right)+(\lambda(n-1)-1) S(n),
\end{aligned}
$$

где $M\left(G F\left(2^{n}\right)\right)$ - сложность умножения в рассматриваемом полиномиальном базисе поля $G F\left(2^{n}\right)$, а $K\left(G F\left(2^{n}\right)\right)$ - сложность возведения в квадрат в том же базисе поля $G F\left(2^{n}\right) . B$ 
случае использования базиса, заданного неприводимьм трехчленом или пятичленом, при $S(n)=O\left(n^{\beta}\right), \beta<2$, справедлива оченка

$$
\begin{aligned}
& I\left(G F\left(2^{n}\right)\right) \leqslant(\lambda(n-1)+v(n-1)) M\left(G F\left(2^{n}\right)\right) \\
& \quad+v(n-1) K\left(G F\left(2^{n}\right)\right)+((2-\beta) \lambda(n-1)+O(1)) S(n) .
\end{aligned}
$$

Если неприводимый трехчлен имеет вид $1+x^{k}+x^{n}, k \leqslant n / 2$, то

$$
I\left(G F\left(2^{n}\right)\right)=O\left(n^{2} \log \log n / \log n\right) .
$$

Действительно, если цепочка $1=a_{0}, \ldots, a_{r}=n-1, r=\lambda(n-1)+v(n-1)-1$, построена бинарным методом, то можно выполнить инвертирование с помощью $\lambda(n-1)+v(n-1)-1$ умножений и $\lambda(n-1)+v(n-1)-1$ операций Фробениуса, из которых $v(n-1)$ являются возведениями в квадрат. Для реализации остальных $\lambda(n-1)-1$ операций Фробениуса выполняем $\lambda(n-1)-1$ операций модулярного умножения, используя (а при программной реализации одновременно вычисляя) необходимые для этого многочлены $x^{2^{a_{i}}} \bmod f$, где $a_{i}$ - такие элементы цепочки, которые в ней на следующем шаге удваиваются. Эти вычисления также выполняются посредством $\lambda(n-1)+v(n-1)-1$ операций модулярного умножения, из которых $v(n-1)$ операций являются возведениями в квадрат. Заметим, что в случае программной, а не схемной реализации при этом уменьшается используемая память, так как вычисление последовательности $x^{2^{a_{i}}} \bmod f$ требует запоминания только одного многочлена во время всего вычисления, а другой многочлен надо помнить во время вычисления промежуточных экспонент $g^{2^{a_{i}}-1} \bmod f$.

Так как возведения в квадрат выполняются отдельно, все используемые операции Фробениуса являются возведениями в степени $2^{a_{i}}$, где последовательность $a_{i}$ растет как геометрическая прогрессия: $a_{i+1} \geqslant 2 a_{i}$. Воспользуясь этим, укажем еще возможность уменьшения сложности вычислений в случае использования базиса, заданного неприводимым трехчленом или пятичленом.

Пусть сложность операции модулярной композиции есть $S(n)=O\left(n^{\beta}\right), \beta<2$, и $k$ таково, что $a_{k}<n^{\beta-1} \leqslant a_{k+1}$. Тогда возведения в степени $2^{a_{i}}, i \leqslant k$, можно выполнить путем повторного возведения в квадрат со сложностью

$$
\sum_{i=1}^{k} O\left(a_{i} n\right)=O\left(a_{k} n\right)=O\left(n^{\beta}\right)
$$

а остальные $(2-\beta) \log _{2} n$ возведений в степени $2^{a_{i}}, i \geqslant k$, выполняем с помощью модулярной композиции. Тогда получаем оценку сложности инвертирования

$$
\begin{aligned}
& I\left(G F\left(2^{n}\right)\right) \leqslant(\lambda(n-1)+v(n-1)) M\left(G F\left(2^{n}\right)\right) \\
& +2 v(n-1) K\left(G F\left(2^{n}\right)\right)+(2(2-\beta) \lambda(n-1)+O(1)) S(n) .
\end{aligned}
$$

При схемной реализации множители 2 в последних слагаемых можно убрать, так как вычисление многочленов $x^{2^{a_{i}}} \bmod f$ выполнять не нужно.

В частности, в случае использования для матричного умножения алгоритма сложности $O\left(n^{3} / \log n\right)$ получаем для сложности программного инвертирования квадратичную оценку (если сложность алгоритма умножения многочленов удовлетворяет оценке $\left.M(n)=O\left(n^{1,4}\right)\right)$. Аналогичную оценку можно получить и для схемной реализации, причем с глубиной $O\left(\log ^{2} n\right)$. Оба этих результата легко получить и без использования 
модулярной композиции, применяя вместо нее упоминавшиеся в 6 схемы сложности $O\left(n^{2} / \log n\right)$ и глубины $\log _{2} n+O(1)$ для вычисления операций Фробениуса (линейных операторов) $g^{2^{k}}$. Однако в случае программной реализации это требует большого расхода памяти, так как матрицы каждой используемой операции Фробениуса надо запоминать, а даже для одной из них это требует $O\left(n^{2}\right)$ памяти. Для выполнения модулярной композиции требуется только $O\left(n^{3 / 2}\right)$ памяти.

Сложность схемы инвертирования можно уменьшить с квадратичной до величины $O\left(n^{2} \log \log n / \log n\right)$, используя схемы возведения в квадрат по модулю неприводимого трехчлена $1+x^{k}+x^{n}, k \leqslant n / 2$, сложности не выше $4 n$ и глубины 2 . Для этого операции возведения в степени $2^{a_{i}}, a_{i} \leqslant n \log _{2} \log _{2} n / \log _{2}^{2} n$, выполняем путем повторного возведения в квадрат со сложностью $O\left(n^{2} \log \log n / \log n\right)$, а остальные операции Фробениуса (их не более $\left.\log _{2} \log _{2} n\right)$ выполняем со сложностью $O\left(n^{2} \log \log n / \log n\right)$. Эта оценка справедлива и для схемной сложности, но глубина схемы получается равной $O\left(n \log \log n / \log ^{2} n\right)$, даже если использовать в качестве неприводимого базисного многочлена $f$ трехчлен.

Известно, что

$$
K\left(G F\left(2^{n}\right)\right) \leqslant 2 M(n)+O(n), \quad M\left(G F\left(2^{n}\right)\right) \leqslant 3 M(n)+O(n),
$$

где $M(n)$ - сложность умножения бинарных многочленов, а если базис задан неприводимым трехчленом или пятичленом, то $K\left(G F\left(2^{n}\right)\right)=O(n)$. В случае существования неприводимого трехчлена степени $n$ обязательно существует трехчлен вида $1+x^{k}+x^{n}$, $k \leqslant n / 2$.

Для уменьшения глубины схемы инвертирования можно вместо стандартной бинарной аддитивной цепочки использовать приведенную в конце $\S 3$ аддитивную цепочку для $n$ глубины $\left\lceil\log _{2} n\right\rceil$ и длины $\lambda(n)+v(n)-1$. Тогда получается схема глубины не более $\left\lceil\log _{2} n\right\rceil\left(D_{M}\left(G F\left(2^{n}\right)\right)+D_{S}(n)\right)$ и сложности

$$
I\left(G F\left(2^{n}\right)\right) \leqslant(\lambda(n-1)+v(n-1)-1)\left(M\left(G F\left(2^{n}\right)\right)+S(n)\right),
$$

где $D_{M}\left(G F\left(2^{n}\right)\right)$ - глубина схемы умножения в рассматриваемом полиномиальном базисе поля $G F\left(2^{n}\right), S(n)$ и $D_{S}(n)$ - верхние оценки сложности и глубины схем для операций Фробениуса в том же базисе. При выборе конкретного значения $n$ и конкретного базиса эту общую оценку можно усилить, оптимизировав схемы для преобразований Фробениуса по глубине и сложности. Например, если

$$
f=1+x^{k}+x^{n}, \quad k \leqslant n / 2,
$$

то сложность и глубину схемы инвертирования можно немного уменьшить, заменив первые $O(\log \log n)$ операций Фробениуса повторным возведением в квадрат.

В практически интересном случае, когда $n$ по порядку не больше нескольких сотен, даже при использовании для умножения метода Карацубы

$$
M\left(G F\left(2^{n}\right)\right)>S(n) .
$$

Поэтому для минимизации сложности инвертирования методом Брауэра вместо бинарной цепочки для $n$ надо, как и в случае нормального базиса, взять цепочку длины $l(n)$. Для практического построения подобной цепочки можно применить $2^{k}$-арный метод (также принадлежащий Брауэру, см. раздел 4.6 .3 в [4]) построения асимптотически минимальной цепочки для числа $n$, в которой все неудваивающие шаги имеют вид

$$
a_{i+1}=a_{i}+a_{l}, \quad a_{l} \leqslant \log _{2} n / \log _{2} \log _{2} n .
$$


Число неудваивающих шагов в этой цепочке равно

$$
l-\lambda(n)=O(\log n / \log \log n),
$$

где $l$ - ее длина. В алгоритме инвертирования этим шагам соответствуют операции Фробениуса $g^{2^{a_{i}}} \bmod f, a_{i} \leqslant \log _{2} n / \log _{2} \log _{2} n$. Так как каждую из них можно выполнить повторным возведением в квадрат, при выборе в качестве $f$ неприводимого трехчлена суммарную сложность этих операций Фробениуса можно оценить как $O\left(n \log ^{2} n / \log ^{2} \log n\right)$, а суммарную глубину можно оценить как $O\left(\log ^{2} n / \log ^{2} \log n\right)$. Для остальных $\lambda(n)$ операций Фробениуса суммарная сложность оценивается как $\lambda(n) S(n)$, а суммарная глубина - как $\lambda(n) D_{S}(n)$. Поэтому справедлива следующая теорема.

Теорема 3. Можно построить схему инвертирования глубины не более

$$
l D_{M}\left(G F\left(2^{n}\right)\right)+\lambda(n-1) D_{S}(n)+O\left(\log ^{2} n / \log ^{2} \log n\right)
$$

и сложности не более

$$
\left.l M\left(G F\left(2^{n}\right)\right)+\lambda(n-1) S(n)+O\left(n \log ^{2} n / \log ^{2} \log n\right)\right),
$$

гдe

$$
l=\lambda(n-1)+\lambda(n) / \lambda(\lambda(n))+O\left(\log n \log \log \log n / \log ^{2} \log n\right)
$$

- длина иепочки для $n-1$, построенной оптимизированным $2^{k}$-арным методом Брауэра (cм. [4]).

В [4] доказано, что в любой цепочке длины $l$ для числа $n$ число неудваивающих шагов не больше $4(l-\lambda(n))$. Если кратчайшая цепочка для $n$ обладает таким свойством, что все неудваивающие шаги имеют вид $a_{i+1}=a_{i}+a_{l}, a_{l} \leqslant \log _{2} n / \log _{2} \log _{2} n$, то указанные выше оценки будут справедливы и для $l=l(n-1)$.

\section{9. Одновременная минимизация аддитивной цепочки по длине и по глубине}

Используя метод Яо [9], докажем, что справедлива следующая теорема.

Теорема 4. Существует аддитивная чепочка для числа $n$ глубины не более $\lambda(n)+2 u$ асимптотически минимальной длины $\lambda(n)+\lambda(n) / \lambda(\lambda(n))+O(\log n \log \log \log n / \log 2 \log n)$. Как следствие, можно построить схему для инвертирования в полиномиальном базисе сложности

$$
I\left(G F\left(2^{n}\right)\right) \leqslant(\lambda(n-1)+(1+o(1)) \lambda(n) / \lambda(\lambda(n)))\left(M\left(G F\left(2^{n}\right)\right)+S(n)\right)
$$

и глубины

$$
D_{I}\left(G F\left(2^{n}\right)\right) \leqslant(\lambda(n-1)+2)\left(D_{M}\left(G F\left(2^{n}\right)\right)+D_{S}(n)\right),
$$

а в нормальном базисе - схему сложности

$$
I\left(G F\left(2^{n}\right)\right) \leqslant(\lambda(n-1)+(1+o(1)) \lambda(n) / \lambda(\lambda(n))) M\left(G F\left(2^{n}\right)\right)
$$

и глубины

$$
D_{I}\left(G F\left(2^{n}\right)\right) \leqslant(\lambda(n-1)+2) D_{M}\left(G F\left(2^{n}\right)\right) .
$$


Действительно, пусть $4 \leqslant n$. Положим

$$
k=\lambda(\lambda(n))-3 \lambda(\lambda(\lambda(n)))+3, \quad s=\lfloor(\lambda(n)-1) / k\rfloor,
$$

и представим $\boldsymbol{n}$ в виде

$$
n=2^{\lambda(n)}+a 2^{k s}+n_{1}, \quad n_{1}=\sum_{i=0}^{s-1} a_{i} 2^{k i}=\sum_{j=1}^{2^{k}-1} j B_{j}, \quad 0 \leqslant a, a_{i}<2^{k},
$$

где

$$
B_{j}=\sum_{a_{i}=j} 2^{k i}
$$

Пусть

$$
M_{j}=\left\{i \mid a_{i}=j\right\}, \quad i=1, \ldots, 2^{k}-1
$$

Тогда

$$
M_{i} \cap M_{j}=\varnothing, \quad i \neq j, \quad \sum_{j=1}^{2^{k}-1}\left|M_{j}\right| \leqslant s
$$

Для непустого множества $M_{j}$ положим

$$
r_{j}=\max \left\{i \mid i \in M_{j}\right\}
$$

Построим цепочку для $\boldsymbol{n}$, оценивая глубину ее элементов. Сначала построим цепочку $1,2,4, \ldots, 2^{\lambda(n)}$. Глубина элемента $2^{i}$ в ней равна $i$. Для вычисления $B_{j}$ (при непустом $M_{j}$ ) строим цепочку, складывая числа $2^{i k}, i \in M_{j}$, в порядке возрастания и помещая каждый очередной член этой цепочки после очередного слагаемого $2^{i k}$ в цепочку степеней двойки. Глубина последнего вставленного элемента не больше $k r_{j}+1$, а число добавленных элементов равно $\left|M_{j}\right|-1, j=1, \ldots, 2^{k}-1$. Общее число всех добавленных элементов не превосходит $s$.

Для вычисления $j B_{j}$ применяем метод множителей: цепочки для $B_{j}$ уже построены, а цепочку для $j B_{j}$ строим при помощи линейной цепочки для $j$ глубины $\left\lceil\log _{2} j\right\rceil$ и длины $\lambda(j)+v(j)-1, j=1, \ldots, 2^{k}-1$. Общее число добавленных элементов не превосходит $3 k 2^{k-1}$. Глубина, с которой вычисляется $j B_{j}$, равна $k r_{j}+1+\left\lceil\log _{2} j\right\rceil$, если $\left|M_{j}\right|>1$, и на 1 меньше, когда $\left|M_{j}\right|=1$, и всегда не превосходит $k\left(r_{j}+1\right)+1$. Аналогично вычисляем $a 2^{k s}$ (в случае, когда $\left.a \neq 0\right)$ с глубиной $k s+\left\lceil\log _{2} a\right\rceil \leqslant \lambda(n)$, добавляя не более $2 k$ элементов.

Пусть числа $j_{i}$ пронумерованы в порядке убывания $r_{j_{i}}$. Складываем числа $j_{i} B_{j_{i}}$ в порядке убывания индекса $i$ (то есть сами числа - в порядке возрастания), тем самым вычисляя $n_{1}$ посредством добавления не более $2^{k}-2$ элементов в цепочку. В силу того, что $r_{j_{i}} \leqslant s-i$, где $i=1, \ldots$, глубина, с которой вычисляется $j_{i} B_{j_{i}}$, не больше $k\left(r_{j_{i}}+1\right)+1 \leqslant$ $k(s-i+1)+1 \leqslant \lambda(n)-i+1$. Поэтому глубина вычисления $n_{1}$ не превосходит $\lambda(n)+1$.

Наконец, вычисляем сумму $n_{1}+a 2^{k s}+2^{\lambda(n)}=n$. Заключительная часть цепочки выглядит так: $\ldots, n_{1}, \ldots, a 2^{k s}, \ldots, 2^{\lambda(n)}, 2^{\lambda(n)}+a 2^{k s}, 2^{\lambda(n)}+a 2^{k s}+n_{1}=n$. Глубина построенной цепочки не превосходит $\lambda(n)+2$, а длина не превосходит

$$
\lambda(n)+s+3 k 2^{k-1}+2 k+2^{k}=\lambda(n)+\lambda(n) / \lambda(\lambda(n))+O(\log n \log \log \log n / \log 2 \log n) .
$$


Используя эту цепочку, методом Брауэра можно построить цепочку для $2^{n}-1$, состоящую из $\lambda(n)+\lambda(n) / \lambda(\lambda(n))+O\left(\log n \log \log \log n / \log ^{2} \log n\right)$ обычных шагов (то есть не удвоений), при этом, если не считать удвоений, глубина такой цепочки не больше $\lambda(n)+2$. Например, применяя метод теоремы 2, можно построить для числа 127 цепочку 1, 2, 4, $5,8,10,15,16,32,48,64,112,127$ длины 12 и глубины 8. Методом из конца §3 строится цепочка $1,2,3,4,7,8,15,16,31,32,63,64,127$ длины 12 и глубины 7. Метод Брауэра для чисел $2^{n}-1$ дает цепочку $1,2,3,4,7,14,28,56,63,126,127$ длины 10 и глубины 9.

Оценка глубины $\lambda(n)+2$ в теореме 2 , вообще говоря, неулучшаема. Легко проверяется, что единственная цепочка для $n=2^{m}-1$ глубины $\lambda(n)+1=m$ имеет вид бинарной цепочки из конца $\S 31,2, \ldots, 2^{i}-1,2^{i}, \ldots, 2^{m-1}-1,2^{m-1}, 2^{m}-1$, и длина ее равна $2 m-2=2 \lambda(n)$.

\section{Список литературы}

1. Agnew G. B., Beth T., Mullin R. C., Vanstone S. A., Arithmetic operations in $G F\left(2^{m}\right)$. J. Crypt. (1993) 6, 3-13.

2. Litow B., Davida G., $O(\log n)$ parallel time finite field inversion. Lecture Notes Computer Sci. (1988) 319, 74-80.

3. Von zur Gathen J., Inversion in finite fields using logarithmic depth. J. Symb. Comput. (1990) 9 , 175-183.

4. Кнут Д., Искусство программирования, т. 2: Получисленные алгоритмы. Вильямс, Москва, 2004.

5. Von zur Gathen J., Nöcker M., Exponentiation in finite fields: theory and practice. Lecture Notes Computer Sci. (1997) 1255, 88-113.

6. Itoh T., Tsujii S., A fast algorithm for computing multiplicative inverses in $G F\left(2^{n}\right)$ using normal basis. Inform. Comput. (1988) 78, 171-177.

7. Takagi N., Yoshiki J., Takagi K., A fast algorithm for multiplicative inversion in $G F\left(2^{n}\right)$ using normal basis. IEEE Trans. Comput. (2001) 50, №5, 394-398.

8. Chang K., Kim H., Kang J., Cho H., An extension of TYT algorithm for $G F\left(\left(2^{n}\right)^{m}\right)$ using precomputation. Inform. Proc. Lett. (2004) 92, 231-234.

9. Yao A. C., On the evaluation of powers. SIAM J. Comput. (1976) 5, 100-103.

10. Von zur Gathen J., Gerhard J., Modern computer algebra. Cambridge Univ. Press, Cambridge, 1999.

11. Лупанов О. Б., Асимптотические оченки сложности управляючих систем. Изд-во МГУ, Москва, 1984.

12. Massey J. L., Omura J. K., Apparatus for finite fields computation. US Patent Application 1986, №4587627.

13. Болотов А. А., Гашков С. Б., О быстром умножении в нормальных базисах конечных полей. Дискретная математика (2001) 13, №3, 3-31.

14. Gao S., von zur Gathen J., Panario D., Shoup V., Algorithm for exponentiation in finite field. $J$. Symb. Comput. (2000) 29, 879-889.

15. Гашков С. Б., Хохлов Р. А., О глубине логических схем для операций в полях $G F\left(2^{n}\right)$. Чебышевский сборник (2003) 4, №4(8), 59-71.

16. Pan V., Complexity of parallel matrix computations. Theor. Comput. Sci. (1987) 54, 65-85. 